\title{
Genome-wide identification and evolution of WNK kinases in Bambusoideae and transcriptional profiling during abiotic stress in Moso bamboo
}

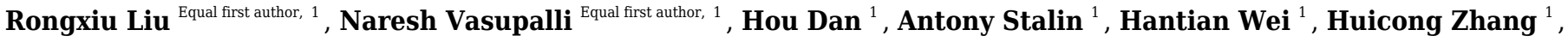 \\ Xinchun Lin ${ }^{\text {Corresp. } 1}$ \\ ${ }^{1}$ Zhejiang A \& F University, State Key Laboratory of Subtropical Silviculture, Lin'an, Zhejiang, China \\ Corresponding Author: Xinchun Lin \\ Email address: Ixc@zafu.edu.cn
}

With No Lysine (WNK) kinases play vital roles in abiotic stress response, circadian rhythms, and regulation of flowering time in rice, Arabidopsis, and Glycine max. However, there are no previous reports of WNKs in the Bambusoideae, although genome sequences are available for diploid, tetraploid, and hexaploid bamboo species. In the present study, we identified 41 WNK genes in five bamboo species and analysed gene evolution, phylogenetic relationship, physical and chemical properties, cis-elements, and conserved motifs. We predicted the structure of PeWNK proteins of moso bamboo and determined the exposed, buried, structural and functional amino acids. Real-time qPCR analysis revealed that PeWNK5, PeWNK7, PeWNK8, and PeWNK11 genes are involved in circadian rhythms. Analysis of gene expression of different organs at different developmental stages revealed that PeWNK genes are tissue-specific. Analysis of various abiotic stress transcriptome data (drought, salt, $\mathrm{SA}$, and $\mathrm{ABA}$ ) revealed significant gene expression levels in all PeWNKs except PeWNK11. In particular, PeWNK8 and PeWNK9 were significantly down- and upregulated, respectively, after abiotic stress treatment. A co-expression network of PeWNK genes also showed that PeWNK2, PeWNK4, PeWNK7, and PeWNK8 were co-expressed with transcriptional regulators related to abiotic stress. In conclusion, our study identified the PeWNKs of moso bamboo involved in circadian rhythms and abiotic stress response. In addition, this study serves as a guide for future functional genomic studies of the WNK genes of the Bambusoideae. 
1 Genome-wide Identification and Evolution of WNK Kinases

2 in Bambusoideae and Transcriptional Profiling During

3 Abiotic Stress in Phyllostachys edulis

4

5

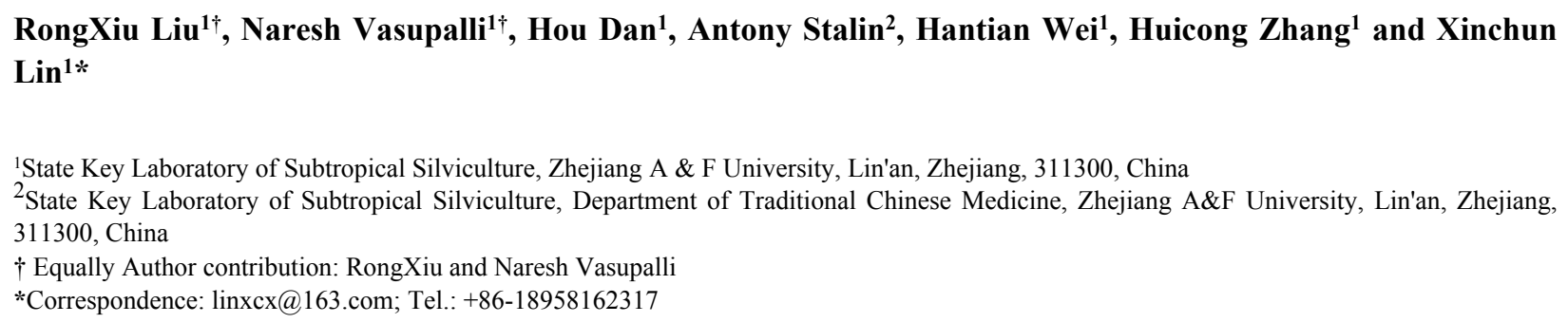

\section{Abstract}

With No Lysine (WNK) kinases play vital roles in abiotic stress response, circadian rhythms, and regulation of flowering time in rice, Arabidopsis, and Glycine max. However, there are no previous reports of WNKs in the Bambusoideae, although genome sequences are available for diploid, tetraploid, and hexaploid bamboo species. In the present study, we identified $41 \mathrm{WNK}$ genes in five bamboo species and analysed gene evolution, phylogenetic relationship, physical and chemical properties, cis-elements, and conserved motifs. We predicted the structure of PeWNK proteins of Phyllostachys edulis and determined the exposed, buried, structural and functional amino acids. Real-time qPCR analysis revealed that PeWNK5, PeWNK7, PeWNK8, and PeWNK11 genes are involved in circadian rhythms. Analysis of gene expression of different organs at different developmental stages revealed that $P e W N K$ genes are tissue-specific. Analysis of various abiotic stress transcriptome data (drought, salt, $\mathrm{SA}$, and $\mathrm{ABA}$ ) revealed significant gene expression levels in all PeWNKs except PeWNK11. In particular, $P e W N K 8$ and $P e W N K 9$ were significantly down- and up-regulated, respectively, after abiotic stress treatment. A co-expression network of $P e W N K$ genes also showed that PeWNK2, PeWNK4, PeWNK7, and PeWNK8 were co-expressed with transcriptional regulators related to abiotic stress. In conclusion, our study identified the PeWNKs of $P$. edulis involved in circadian rhythms and abiotic stress response. In addition, this study serves as a guide for future functional genomic studies of the $W N K$ genes of the Bambusoideae.

Keywords: WNK, Phyllostachys edulis, Gene expression, Abiotic stress

\section{Introduction}

Protein kinase is a large superfamily of enzymes known to phosphorylate the threonine, tyrosine, and serine residues of target proteins (Kumar et al., 2020). They constitute about $4 \%$ of the Arabidopsis thaliana proteome and are involved in various functions such as development, cell cycle and signal transduction (Manuka et al., 2019; Manuka et al., 2015; Wang et al., 2008). A unique subfamily of serine/threonine protein kinases related to the STE20/PAK-like family is called With-No-Lysine (WNK) kinases and is found only in multi-cellular organisms (Kumar et al., 2011; Xu et al., 2000). The WNK kinases contain a conserved lysine residue in the subdomain 
41 II within the N-terminal domain, which is essential for ATP binding. However, this conserved

42

43

44

45

46

47

48

49

50

51

52

53

54

55

56

57

58

59

60

61

62

63

64

65

66

67

68

69

70

71

72

73

74

75

76

77

78

79

80 lysine residue in the active site is absent in the WNK subdomain II (Xu et al., 2000). Moreover, the lysine in subdomain-I is involved in kinase phosphorylation, and it is the characteristic feature of the WNK family (McCormick \& Ellison. 2011).

In plants, $W N K$ genes are involved in physiological functions such as maintenance of circadian cycle, root architecture, signal transduction, response to abiotic stress, and flowering time by affecting photoperiod (Kahle et al., 2006; Urano et al., 2015; Urano et al., 2012; Wang et al., 2010). Currently, $11 W N K s$ are known in $A$. thaliana and nine $W N K s$ in rice, but only a few genes have been well studied (Manuka et al., 2015). For example, AtWNK1 phosphorylates APRR3 protein, the part of APRR1/TOC1 quintet associated with the clock, to regulate circadian rhythms (Nakamichi et al., 2002). At the same time, the involvement of AtWNK2, AtWNK4, and AtWNK6 in circadian rhythms has also been reported (Nakamichi et al., 2002). Similarly, OsWNK1 shows a rhythmic expression profile under circadian and diurnal conditions and responds to abiotic stress in rice (Kumar et al., 2011).

Furthermore, a knock-out study has demonstrated the importance of $A t W N K 8$ in abiotic stress (Zhang et al., 2013) and overexpression of AtWNK9 increases drought tolerance through the ABA signaling cascade (Xie et al., 2014). In addition, nine $W N K(1-9)$ have been identified in rice that exhibits differential transcriptional regulation for different abiotic stresses such as heat, cold, salt, and drought (Manuka et al., 2015). At the same time, overexpression of OsWNK9 enhances the tolerance to salt, drought, and arsenite in A. thaliana (Manuka et al., 2021; Xu et al., 2000). Similarly, root-specific GmWNK1 in Glycine max regulates root system architecture and stress response via an ABA-dependent signaling pathway (Rodan \& Jenny. 2017). At the same time, overexpression of GmWNK1 in A. thaliana showed tolerance towards osmatic and salt stress (Wang et al., 2011). In addition, a total of $114 W N K s$ were identified from eight fruit tree species. It was predicted that $P p W N K . A 2$ and $P p W N K$.E3.1 genes might be related to early fruit development, while PpWNK.A1 is likely associated with fruit ripening (Cao et al., 2019).

Bamboos (Bambusoideae) are among the fastest-growing plants globally, and Phyllostachys edulis (moso bamboo) is the most widespread bamboo species in China and has high economic value as edible shoots, timber, and pulp (Choudhury et al., 2012). Bamboo can be divided into four monophyletic lineages based on the level of ploidy: diploid herbaceous bamboo, tetraploid temperate and neotropical woody bamboo, and hexaploid paleotropical woody bamboo. Recently, Zhao et al. (2018) reported the chromosome level P. edulis (temperate tetraploid woody bamboo) whole-genome sequence. At the same time, Guo et al. (2019) reported the draft genome sequences of Olyra latifolia and Raddia guianensis (diploid herbaceous bamboo), Guadua angustifolia (tetraploid neotropical woody bamboo) and Bonia amplexicaulis (hexaploid paleotropical woody bamboo). Due to climate change, naturally growing bamboo species were subjected to different kinds of abiotic stress. Recently Liu et al. (2019) reported that the P. edulis yield and the quality of winter shoots were severely affected by abiotic stress conditions. Therefore, studying the genes involved in abiotic stress in bamboo species is helpful to develop better adapted genetically modified bamboo plants to the changing environment. The availability of the chromosome level

Peer] reviewing PDF | (2021:08:65078:2:0:NEW 25 Nov 2021) 
81

82

83

84

85

86

87

88

89

90

91

92

93

94

95

96

97

98

99

100

101

102

103

104

105

106

107

108

109

110

111

112

113

114

115

116

genome of $P$. edulis, draft genome sequences of other bamboo species, and various transcriptomic data from tissues provide the opportunity for genome-wide analysis WNK genes (Guo et al., 2019; Zhao et al., 2018). In this study, we identified $41 \mathrm{WNK}$ genes belonging to the five bamboo species. Then, we analysed the physicochemical properties, protein structure, and evolution of the WNKs of the Bambusoideae. We also analysed the expression of PeWNKs genes in different tissues, the response to abiotic stress, and the co-expression network. The present study results provide a basis for the functional analysis of $W N K$ genes in P. edulis.

\section{Materials \& Methods}

\section{Plant materials}

P. edulis seeds used for transcriptomic data were collected in Linchuan County, Guangxi Zhuang Autonomous Region, China. For qPCR analysis, $P$. edulis leaves were collected from the Cuizhu Garden of Zhejiang Agriculture and Forestry University. Samples were collected every four hours, from 6 AM on April 25, 2021, to 48 hours.

\section{Identification of $W N K$ Genes from $P$. edulis Genome Databases}

The $W N K$ genes of $A$. thaliana and rice were downloaded from the Phytozome (https://phytozome.jgi.doe.gov/pz/portal.html). We used the genome database of $P$. edulis and transcriptomic data (Zhao et al., 2018) to identify $W N K$ family genes through the local BLAST analysis. At the same time, other $W N K$ genes of the Bambusoideae were isolated from draft sequences of the herbaceous diploid bamboo species $O$. latifolia and $R$. guianensis and the tetraploid and hexaploid woody species G. angustifolia and B. amplexicaulis (Guo et al., 2019). The candidate genes obtained were verified against the NCBI database (https://www.ncbi.nlm.nih.gov/). The amino acid sequences of $W N K$ genes were aligned to confirm conserved regions. The sequences without a complete reading frame and conserved domain were removed.

\section{Physicochemical Properties, Phylogenetic Tree and Motif Analysis of WNK Genes}

The amino acid number, molecular weight, and isoelectric point of PeWNK proteins were calculated using the online software ExPASy (https://www. ExPASy.org/). The phylogenetic tree was constructed using the maximum-likelihood method with MEGA-X (Kumar et al., 2018). The conserved domains of plant species A. thaliana, Glycine max, Oryza sativa, Zea mays, P. edulis, R. guianensis, O. latifolia, G. angustifolia and B. amplexicaulis were used to construct the phylogenetic tree. A bootstrap value of 1,000 replicates was calculated to evaluate the statistical significance of clade level relationships. Subsequently, the phylogenetic tree for WNKs was imported into the ITOL server (http://itol.embl.de/). The conserved motifs were identified using the MEME server and visualized in TBtools (Chen et al., 2020).

Protein Secondary and Tertiary Structure of PeWNK Genes 
117

118

119

120

121

122

123

124

125

126

127

128

129

130

131

132

133

134

135

136

137

138

139

140

141

142

143

144

145

146

147

148

149

150

151

152

153

The secondary structures of the WNK proteins of $P$. edulis were predicted through the online website SOPMA (https://npsa-prabi.ibcp.fr/npsa sopma.html) with the default parameters of four conformational states (helix, sheet, turn, coil) and similarity threshold eight. The tertiary structures of the WNK proteins of $P$. edulis were predicted using the Modeller tool with the help of the Consurf server (Berezin et al., 2004). The models of the proteins were built based on the 'ConSeq' mode and the given selected parameters were used to build the multiple sequence alignments. The homologs were taken from the UniProt database and CS-BLAST was used as the algorithm for homolog search (CSI-BLAST E-value: 0.0001; No. of CSI-BLAST Iterations: 3; maximal percentage ID between sequences: 95; minimal percentage ID for homologs: 35; 150 sequences querying the list of homologs for retrieval. For phylogenetic tree analysis, Neighbor-Joining with ML distance algorithm was used. Bayesian computational calculation and best-fit model of substitution for proteins were used to calculate the conservation scores.

\section{Analysis of Cis-acting Element}

We retrieved the upstream sequence region $(2 \mathrm{~Kb})$ of the $W N K$ genes from the genome database to analyse the cis-acting elements. The retrieved sequences were analysed using the PlantCARE program (http://bioinformatics.psb.ugent.be/webtools/plantcare/html/) to identify the putative cis-acting elements. The cis-elements related to ABA, GA, SA and circadian rhythms were visualized through TBtools (Chen et al., 2020)

\section{The PeWNK Gene Expression in Different Tissues}

Transcriptome data of 26 different tissues of $P$. edulis were obtained from the NCBI Short Read Archive database (SRX2408703) (Zhao et al., 2018) and used for tissue expression studies. The FPKM values of the $W N K$ genes of $P$. edulis were used to develop a heat map using TBtools (Chen et al., 2020).

\section{Expression Analysis of PeWNK Genes in Response to Abiotic Stress}

Thirty-day old equal height $P$. edulis seedlings were used for abiotic stress treatment. Seedlings were treated with 25\% polyethylene glycol (PEG), $200 \mu \mathrm{M}$ Abscisic acid (ABA), $1 \mathrm{mM}$ salicylic acid (SA) (unpublished) and $200 \mathrm{mM}$ sodium chloride ( $\mathrm{NaCl}$ ) (Yang et al., 2010) nutrient solution for $3 \mathrm{~h}$ and $24 \mathrm{~h}$, respectively. Total RNA was isolated from young leaves and RNAseq data were generated on the Illumina platform (pair-end reads) in three biological and technical replicates (unpublished data). The adapter sequences and low-quality reads were removed and the high-quality reads were mapped to the reference genome sequence using the Hisat 2 tool. FPKM values of the RNAseq data were developed and used to generate graphs.

\section{Real-time qPCR Analysis}

Total RNA from leaf samples was isolated using Trizol reagent. According to the manufacturer's instructions, cDNA was synthesised using the PrimeScript RT reagent kit with gDNA Eraser (TaKaRa, Shiga, Japan). The 2XNovoStart SYBR qPCR SuperMix Plus (novoprotein, Suzhou, China) was used for qRT-PCR amplification in a real-time PCR instrument 
154 (BioRad, USA). The qPCR reaction conditions are as follows: initial denaturation $95^{\circ} \mathrm{C}$ for $5 \mathrm{~min}$,

155

156

157

158

159

160

161

162

163

164

165

166

167

168

169

170

171

172

173

174

175

176

177

178

179

180

181

182

183

184

185

186

187

188

189

190

191

followed by 40 cycles of $30 \mathrm{~s}$ at $94^{\circ} \mathrm{C}, 30 \mathrm{~s}$ at $60^{\circ} \mathrm{C}$, and $30 \mathrm{~s}$ at $72^{\circ} \mathrm{C}$. A melting curve was included from 65 to $95^{\circ} \mathrm{C}$ to check amplification specificity. The $2-\Delta \Delta \mathrm{Ct}$ method was used to determine the relative expression levels. In addition, $N T B$ was used as a reference gene in $P$. edulis according to previous studies (Zhao et al., 2019). The qPCR primers for the PeWNK genes used for gene expression analysis are listed in Table S1.

\section{Co-expression Analysis of $P e W N K$ genes}

We submitted the PeWNK genes to the BambooNET (http://bioinformatics.cau.edu.cn/bamboo/index.html) and acquired the co-expression network data.

\section{Results}

\section{Identification of the Bambusoideae WNK Genes}

The genome database of $P$. edulis and the draft genomes of $R$. guianensis, $O$. latifolia, $G$. angustifolia, and $B$. amplexicaulis were used to find the $W N K$ candidate genes in the Bambusoideae. In addition, the $W N K$ genes of $A$. thaliana and rice were downloaded from Phytozome and used as reference genes to identify the $W N K$ genes of the Bambusoideae through the local BLASTP. The sequences containing the serine/threonine-protein kinase domain are referred to as Bambusoideae WNK genes (PeWNKs, RguWNKs, OlaWNKs, GanWNKs, and BamWNKs) (File S1). A total of 11 WNK genes of P. edulis (PeWNK1-11) and 30 WNK genes of the other four bamboo species were identified. The WNK proteins of the Bambusoideae range from 257 to 1905 amino acids, of which RguWNK1 is the smallest and PeWNK8 is the largest. At the same time, the molecular weight is 29047.42 and $157,857.24$, respectively. Moreover, the isoelectric point and instability index are 4.56 to 6.74 and 29 to 59.63 , respectively. In addition, the aliphatic index and the grand average of hydropathicity are 19.42 to 95.95 and -0.647 to 0.977 , respectively (Table S2). Furthermore, we identified that $P e W N K$ genes were located on nine scaffolds, with scaffolds 4 and 10 containing two genes, whereas the remaining scaffolds contained only one gene (Fig. S1).

\section{Evolution of $W N K$ gene Family}

To understand the evolution of $W N K$ genes, a total of $78 W N K$ genes (AtWNKs, GmWNKs, OsWNKs, ZmWNKs, PeWNKs, BamWNKs, GanWNKs, OlaWNK, and RguWNKs) were used to construct the phylogenetic tree (File S1). The highly aligned peptide sequences were used to generate a phylogenetic tree using the maximum likelihood method with 1,000 bootstrap replicates (Fig. 1). The $W N K s$ were mainly divided into three clades, namely clade I, II and III. In addition, clade III was divided into clades IIIA and IIIB and clade III has more genes than clades I and II. Additionally, all clades were supported by high bootstrap values. Based on the topological structure, the evolution of $W N K$ genes was clearly divided between monocots and dicots in the phylogenetic tree. In clades, I, II, IIIA, and IIIB, monocot and dicot $W N K$ genes were divided into two sub-branches with higher bootstrap values. These results suggest that WNKs were present 
192 before the divergence of monocot and dicot plants. Moreover, the OlaWNKs and RguWNKs of 193 herbaceous bamboo were also divided into sub-branches compared with the other woody bamboo 194 species. This suggests that WNKs evolved separately after polyploidisation in the Bambusoideae 195 (Fig. 1).

196 The evolution of plant species is driven by polyploidisation, including in the Bambusoideae 197 (Ramakrishnan et al., 2020). In the phylogenetic tree, the WNKs of diploid and polypoid bamboo 198 species were also separated by sub-braches. Moreover, the copy number of $W N K s$ was increased 199 in the tetraploid $P$. edulis and hexaploid B. amplexicaulis compared to the diploid bamboo species 200

201

202

203

204

205

206

207

208

209

210

211

212

213

214

215

216

217

218

219

220

221

222

223

224 $O$. latifolia and $R$. guianensis. In contrast, the copy number of $W N K s$ is surprisingly lower in the tetraploid $G$. angustifolia than in the diploid bamboo species. Furthermore, we analysed the evolution of specific domains in WNKs between dicot and monocot plants (Fig. 1). Using the MEME server, we identified ten conserved motifs in the WNKs proteins. With few exceptions, most WNKs in all three clades contain all ten domains in the same serial order. RguWNKI in clade II and GmWNK4 in clade III contain the least number of six domains. BamWNK3 in clade I, on the other hand, has 17 domains, with domains $1,2,3,5,7,8$, and 10 were duplicated. In addition, the starting domain nine is absent in most of the monocot groups of clade I. In contrast, in clade II, the last two domains 7 and 10 are missing in half of the bamboo WNKs.

\section{Cis-acting Elements Responsive to Abiotic Stress and Circadian Rhythm}

Cis-acting elements affect genes involved in the stress response. Hence, studying the cisacting elements in the promoter region helps to understand the role of $W N K$ genes in the stress response. Therefore, we analysed the putative cis-elements in the $2 \mathrm{~kb}$ region upstream of the translational start site of WNK genes in both monocot (OsWNKs, PeWNKs, BamWNKs, GanWNKs, $O l a W N K$, and $R g u W N K s$ ) and dicot (AtWNKs and GmWNKs) plants (File S2). Among them, we focused on exploring the ABA, GA, SA and circadian rhythm responsive elements, and there are several cis-elements associated with them in WNKs. For example, ABA-responsive elements (ABREs) are present in 61 genes, including all $11 \mathrm{PeWNK}$ genes (File S3). We, therefore, hypothesise that ABA stress responses regulate most $W N K$ genes. Moreover, the GA responsive GARE-motif, P-box, and TATA-box elements are present in the promoter regions of 21, 18, and $71 W N K$ genes, respectively. Similarly, SA responsive element TCA is present in the promoter region of $24 W N K$ genes. Interestingly, $12 W N K$ genes also have cis-acting elements associated with circadian control (Fig. 2). Further, GC-motif and SP1 are present in some of the monocot $W N K$ and $G m W N K$ genes but absent in Arabidopsis (Fig. S2).

\section{Prediction of the Protein Structure of $P$. edulis WNK}

225

226

227

228

229

230

The secondary structure of the protein plays an essential role in constructing the tertiary structure of the protein and its normal function. It mainly consists of hydrogen bonds and the primary forms include $\alpha$-helix, $\beta$-turn, random curling, etc. The secondary structure of 11 P. edulis WNK proteins was predicted using the online website SOPMA (Table 1). It can be seen that the WNK protein of $P$. edulis has a relatively similar protein secondary structure. Modeller9.19 software was used to predict the tertiary structure of 11 identified WNK proteins of $P$. edulis 
231

232

233

234

235

236

237

238

239

240

241

242

243

244

245

246

247

248

249

250

251

252

253

254

255

256

257

258

259

260

261

262

263

264

265

266

267

268

(Ashkenazy et al., 2016). Furthermore, we compared and analyzed the domains and motifs of PeWNK1 with human_WNK3, GmWNK1, OsWNK9 and AtWNK1 (Fig. 3). The PeWNK1 sequence was similar to all other previously published WNKs genes (Manuka et al., 2018). PeWNK1 has an $\mathrm{N}$-terminal protein kinase domain divided into 12 subdomains. In addition, an activation loop (Aloop), an autoinhibitory conserved domain-containing FXF motif, the 'IIHRDLKCDNIFI' motif in subdomain VIb and the 'GTPEFMAPE' motif in subdomain VIII were conserved (Fig. 3). Besides, we compared the eleven $W N K$ genes from $P$. edulis in which all these A-loops and motifs were conserved (Fig. S3), and we also detected that these A-loops and motifs were conserved in all monocot and dicot plants used in this study (File S1). Moreover, we also analysed the phosphorylation sites of the PeWNK proteins and identified that, except for PeWNK9, all other WNKs contained the phosphorylation sites (Fig. 4 and Fig. S3).

Furthermore, all PeWNK protein sequences were compared with known WNK proteins in the Uniprot database using ConSurf domain analysis (Ashkenazy et al., 2010; Celniker et al., 2013). Based on the phylogenetic relationship between the homologous sequences of WNK, the conserved regions of amino acids were identified. For instance, the conserved domain region of PeWNK1 is shown in the colour magenta (Fig. 4). The remaining conserved domains of the ten PeWNK proteins are listed in a supplementary file (File S4). As mentioned in Fig. 3, most of the amino acids in the activation loop, autoinhibitory domain (FPF), and kinase domain are located in the conserved region. We also identified the exposed, buried, functional and structural (e, b, f, s) residues/amino acids in the PeWNKs. All functional residues are the exposed residues, while all structural residues are buried (Fig. 4).

\section{PeWNK Genes Response to Circadian Rhythms}

$W N K$ genes have been previously reported to be involved in circadian rhythms (Kumar et al., 2011; Nakamichi et al., 2002). Therefore, we collected leaf samples of P. edulis every four hours starting from $6 \mathrm{AM}$ up to $48 \mathrm{~h}$ and conducted qPCR experiments to identify the PeWNK genes of $P$. edulis involved in circadian rhythms. The results showed that among the $11 \mathrm{PeWNK}$ genes of $P$. edulis, PeWNK5, PeWNK7, PeWNK8, and PeWNK11 follow circadian rhythms (Fig. 5). The $P e W N K 7, P e W N K 8$, and $P e W N K 11$ genes show a clear circadian expression pattern in the morning, with a peak forming every 0 and $4 \mathrm{~h}(6$ and $10 \mathrm{AM})$. In contrast, the expression pattern of PeWNK5 follows a $12 \mathrm{~h}$ cycle. After $0 \mathrm{~h}$ in the morning, the expression drops to a very low level at $4 \mathrm{~h}$ and increases again at 8 and $12 \mathrm{~h}$ (2 and 6 PM) (Fig. 5).

\section{Expression Profile of PeWNK Genes in Different Tissues}

To elucidate the expression profiles of PeWNKs in different tissues, we developed a heatmap using transcriptomic data from 26 different tissues at different developmental stages, as mentioned by Zhao et al. (2018). The heatmap indicates that some $P e W N K$ genes have high expression in specific tissues. For example, the expression patterns of $P e W N K 10$ were very high in the middle and lower portion of the $3 \mathrm{~m}$ shoot, while the expression in the other tissues was comparatively low. In addition, PeWNK7 was expressed in the rhizome, whereas PeWNK6 and PeWNK1 were

Peer) reviewing PDF | (2021:08:65078:2:0:NEW 25 Nov 2021) 
269

270

271

272

273

274

275

276

277

278

279

280

281

282

283

284

285

286

287

288

289

290

291

292

293

294

295

296

297

298

299

300

301

302

303

304

305

306

mainly expressed in the leaf. Interestingly, the expression of $P e W N K$ genes was relatively low in the rhizome bud (budR), lower bud, and top 3m shoot (Fig. 6).

\section{Response of PeWNK Genes under Abiotic Stress treatments}

We analysed the transcriptomic data to investigate further the characteristics of $P e W N K$ gene expression in $P$. edulis seedlings under drought, salt, SA and ABA treatments. The analyses showed that $P e W N K$ genes responded differently at $3 \mathrm{~h}$ and $24 \mathrm{~h}$ after exposure to drought, salt and hormone stress. In this study, the genes with two-fold differences were considered to be differentially expressed compared with the control (Wang et al., 2020). Among all PeWNK genes, the expression of $P e W N K 9$, in particular, was significantly up-regulated after abiotic stress treatments (Fig. 7). Under PEG, $\mathrm{NaCl}$ and $\mathrm{ABA}$ treatment, the relative expression of $P e W N K 9$ was up-regulated 146, 117, and 307 times respectively, after $24 \mathrm{~h}$ compared with the control. Similarly, the relative expression of $P e W N K 4$ was up-regulated by 2.2-8.2 times of control after $3 \mathrm{~h}$ in all treatments. Further, the relative expression of PeWNK7 and PeWNK8 was significantly downregulated after $3 \mathrm{~h}$ in all treatments. After 24 hours of treatment with SA, the expression of $\mathrm{PeWNK} 7$ was up-regulated to 2.6 times and the expression of $P e W N K 8$ was downregulated to 0.45 times of control (Fig. 7).

After $3 \mathrm{~h}$ of treatment with SA, the relative expression of $P e W N K 1$ was downregulated to 0.23 times of control. Similarly, the expression of $P e W N K 2$ was significantly downregulated after $3 \mathrm{~h}$ of PEG, SA, ABA and $24 \mathrm{~h}$ of $\mathrm{NaCl}$ treatment. Likewise, the expression of PeWNK5 and PeWNK6 was downregulated to 0.4 times after $3 \mathrm{~h}$ of SA treatment. While the expression of PeWNK6 was up-regulated to 2.5 times of control after $3 \mathrm{~h}$ of ABA treatment. The expression of $P e W N K 10$ was significantly up-regulated after both $\mathrm{NaCl}$ treatment and $24 \mathrm{~h} \mathrm{SA}$ treatment. At the same time, expression was downregulated to 0.4 times of control after $3 \mathrm{~h}$ treatment with PEG and $24 \mathrm{~h}$ treatment with ABA. The expression levels of PeWNK11 are too low for analysis (Fig. 7).

\section{Co-expression Analysis of PeWNK Genes}

A co-expression network has been successfully applied to identify the transcription factors or regulators in many plant species (Bishop et al., 2020; Gao et al., 2020; Yang et al., 2017). To determine the regulators of PeWNK genes, we used the BambooNET database. The 11 PeWNK genes were searched for transcriptional regulators in the BambooNET database. PeWNK8 (PH02Gene03413.t1) is co-expressed with 17 genes, including GRAS family transcription factor and F-box protein 2 (Fig. 8). Interestingly, both genes have been reported to be associated with abiotic stress. Similarly, both PeWNK2 (PH02Gene17877) and PeWNK4 (PH02Gene23702) were co-expressed with an F-box family protein (PH02Gene00258). Furthermore, PeWNK7 (PH02Gene03314) is co-expressed with the PEBP (phosphatidylethanolamine-binding protein) family protein and the myb domain protein 48 (File S5). These two proteins are involved in the suppression of flowering and circadian rhythms, respectively.

\section{Discussion}


307

308

309

310

311

312

313

314

315

316

317

318

319

320

321

322

323

324

325

326

327

328

329

330

331

332

333

334

335

336

337

338

339

340

341

342

343

344

345

346

Bamboo is one of the fastest-growing perennial plants and has the longest vegetative stage before flowering (Liu et al., 2019; Ramakrishnan et al., 2020). However, the mechanisms involved in abiotic stress during bamboo growth are poorly understood. WNK genes, which belong to the serine/threonine protein kinases of the STE20/PAK-like subfamily (Manuka et al., 2015) play an essential role in regulating plant salt tolerance and osmotic stress by coordinating ion channels and signal transduction during the transportation process (Kahle et al., 2006; Wang et al., 2010). In addition, $W N K$ genes are also involved in circadian rhythms (Nakamichi et al., 2002). To date, WNK genes have been identified in Arabidopsis, rice, soya bean, and fruit trees (Cao et al., 2019; Kumar et al., 2011; Wang et al., 2008). However, the identity and function of $W N K s$ in bamboo, including $P$. edulis, have not yet been identified. In this study, we identified $W N K$ genes in diploid and polyploid bamboo species and investigated the evolution of $W N K s$ between monocot and dicot plants. Further, we identified the protein structure, response to abiotic stress, tissue-specific expression, and co-expression analysis of PeWNK genes in P. edulis.

We identified a total of 41 WNK genes from the available bamboo genome database and investigated their gene evolution, physical and chemical properties, and conserved motifs. The putative amino acid lengths of WNKs from Rice, G. max, and Populus trichocarpa range from 328-705, 480-738 and 297-739 amino acids, respectively (Manuka et al., 2015; Wang et al., 2010). At the same time, human WNK1 has a length of 2382 amino acids (VerõÂssimo \& Jordan. 2001). In our study, the length of the amino acids of WNK of diploid bamboo is 257-702, that of tetraploid bamboo is 285-1905, and that of hexaploid bamboo is 290-739. These results suggest that the amino acid lengths of diploid, hexaploid and tetraploid GanWNKs are similar to those of rice and G. max. Interestingly, the amino acid length of the four PeWNK genes in P. edulis ranges from 1771-1905, which is almost the size of human WNKs and three times longer than OsWNK.

PeWNKs have the N-terminal protein kinase domain, which has the altered lycine residue in the Gly-X-Gly-X-X-Lys-X-Val motif of subdomain I instead of Gly-X-Gly-X-X-Gly-X-Val. In addition, the $W N K$ genes of higher plants were divided into three clades. These results are consistent with previous findings in plants and animals (Manuka et al., 2015; Xu et al., 2000). Moreover, the distribution of conserved motifs was similar among WNK proteins in the same clade. These results and phylogenetic analysis support the reliability of clade classification and the similar functions of proteins in the same clade. Moreover, the number of genes in the gene families increased with the duplication events and polyploidization (De Grassi et al., 2008; Li et al., 2020). The copy number of $W N K s$ was increased in the tetraploid $P$. edulis and hexaploid B. amplexicaulis compared to the diploid bamboo species $O$. latifolia and $R$. guianensis. In contrast, the copy number of $W N K S$ is lower in the tetraploid G. angustifolia than in the diploid bamboo species. These results might be due to low coverage, poor sequencing, and incomplete genome database.

Tissue-specific expression analysis of $O s W N K$ genes in rice revealed that most $O s W N K$ genes are more highly expressed in roots than in other tissues, indicating the role of $O s W N K s$ in root formation and architecture (Manuka et al., 2015). In Arabidopsis, AtWNK8 is mainly expressed in the hypocotyl, primary root, and pistil (Zhang et al., 2013). At the same time, all other AtWNK genes (except $A t W N K \sigma$ ) are expressed in different tissues and organs at different developmental 
347 stages (Wang et al., 2008). In the fruit tree Prunus persica, gene expression analysis revealed that $348 P p W N K . A 1$ is probably involved in fruit ripening, while PpWNK.A2 and PpWNK.E3.1 are

349

350

351

352

353

354

355

356

357

358

359

360

361

362

363

364

365

366

367

368

369

370

371

372

373

374

375

376

377

378

379

380

381

382

383

384

385

386 associated with early fruit development (Cao et al., 2019). In contrast to rice OsWNKs, tissuespecific expression analysis of $P e W N K$ genes in our study shows that most $P e W N K$ genes are expressed only in a particular tissue at a specific plant height, indicating diverse roles in different developmental stages of the tissues.

Various abiotic stress conditions severely affect $P$. edulis yield and the quality of winter shoots (Liu et al., 2019). Protein kinases in plants play a crucial role in stress-induced signal transduction pathways (Kundan Kumar et al., 2013). Our results showed that all PeWNK genes responded to abiotic stress, except PeWNK11. A T-DNA knock-out mutant study showed that AtWNK8 was induced after salt and sorbitol stress, and disruption of AtWNK8 enhances tolerance to $\mathrm{NaCl}$ and osmotic stress (Zhang et al., 2013). Moreover, overexpression of OsWNK9 increases tolerance to salt, drought, and arsenite in transgenic Arabidopsis plants (Manuka et al., 2019; Manuka et al., 2021). Phylogenetic analysis of the gene family shows that AtWNK8 and OsWNK9 are closely related to $P e W N K 7, P e W N K 8$, and $P e W N K 9$. Our study also provided evidence that the expression of $P e W N K 9$ was significantly increased after all abiotic stress treatments. In contrast, the expression of $P e W N K 8$ significantly decreased considerably after $3 \mathrm{~h}$ of PEG, $\mathrm{NaCl}$ and SA treatments. Similarly, the $O s W N K 1$ gene was up-regulated after drought and cold stress and downregulated after salt stress (Kumar et al., 2011). Both PeWNK1 and PeWNK2 were similar to $O s W N K 1$ and both were significantly downregulated after all abiotic stresses studied. These results suggest that these proteins have similar functions and are predominantly involved in abiotic stress response.

In addition, our co-expression network analysis also revealed the relationship between abiotic stress genes and $P e W N K$ genes. In this study, PeWNK8 was found to be co-expressed with transcription factor GRAS and F-box protein 2. The transcription factor OsGRAS23 from rice is involved in drought stress response, and the transcription factor GRAS from Vitis amurensis induces abiotic stress tolerance in Arabidopsis (Xu \& Zhang. 2015; Yuan et al., 2016). Similarly, an F-box protein MAX2 regulates drought tolerance in Arabidopsis (Bu et al., 2014). Interestingly, $P e W N K 8$ was downregulated after $\mathrm{PEG}, \mathrm{NaCl}$ and $\mathrm{SA}$ treatments, indicating its involvement in the abiotic stress response.

\section{Conclusions}

In the present study, we identified $41 W N K$ genes in five Bambusoideae species and analyzed the conserved motifs, domains, cis-acting elements, and tissue-specific expression studies. The qRT-PCR analysis revealed that PeWNK5, PeWNK7, PeWNK8, and PeWNK11 are involved in circadian rhythms. Transcriptome analysis of different abiotic stresses and co-expression analysis also revealed that $P e W N K 8$ and $P e W N K 9$ are involved in abiotic stress response. Thus, these genes can be used as good candidates for the production of genetically modified and economically important bamboo plants. 
387

388

389

390

391

392

393

394

395

396

397

398

399

400

401

402

403

404

405

406

407

408

409

410

411

412

413

414

415

416

417

418

419

420

421

422

423

424

425

426

427

428

429

430

\section{Acknowledgments}

This work was supported by grants from the Natural Science Foundation of Zhejiang Province (LZ20C160002), the National Natural Science Foundation of China (31971735), and the State Key Laboratory of Subtropical Silviculture (ZY20180203).

Conflicts of Interest: The authors declare no conflict of interest.

\section{References}

Ashkenazy H, Abadi S, Martz E, Chay O, Mayrose I, Pupko T, Ben-Tal N. 2016. ConSurf 2016: an improved methodology to estimate and visualize evolutionary conservation in macromolecules. Nucleic Acids Research 44:W344-350. 10.1093/nar/gkw408

Ashkenazy H, Erez E, Martz E, Pupko T, Ben-Tal N. 2010. ConSurf 2010: calculating evolutionary conservation in sequence and structure of proteins and nucleic acids. Nucleic Acids Research 38:W529-533. 10.1093/nar/gkq399

Berezin C, Glaser F, Rosenberg J, Paz I, Pupko T, Fariselli P, Casadio R, Ben-Tal N. 2004. ConSeq: the identification of functionally and structurally important residues in protein sequences. Bioinformatics 20:1322-1324. 10.1093/bioinformatics/bth070

Bishop EH, Kumar R, Luo F, Saski C, Sekhon RS. 2020. Genome-wide identification, expression profiling, and network analysis of AT-hook gene family in maize. Genomics 112:12331244. 10.1016/j.ygeno.2019.07.009

Bu Q, Lv T, Shen H, Luong P, Wang J, Wang Z, Huang Z, Xiao L, Engineer C, Kim TH, Schroeder JI, Huq E. 2014. Regulation of drought tolerance by the F-box protein MAX2 in Arabidopsis. Plant Physiology 164:424-439. 10.1104/pp.113.226837

Cao S, Hao P, Shu W, Wang G, Xie Z, Gu C, Zhang S. 2019. Phylogenetic and Expression Analyses of With-No-Lysine Kinase Genes Reveal Novel Gene Family Diversity in Fruit Trees. Horticultural Plant Journal 5:47-58. 10.1016/j.hpj.2019.01.006

Celniker G, Nimrod G, Ashkenazy H, Glaser F, Martz E, Mayrose I, Pupko T, Ben-Tal N. 2013. ConSurf: Using Evolutionary Data to Raise Testable Hypotheses about Protein Function. Israel Journal of Chemistry 53:199-206. 10.1002/ijch.201200096

Chen C, Chen H, Zhang Y, Thomas HR, Frank MH, He Y, Xia R. 2020. TBtools: An Integrative Toolkit Developed for Interactive Analyses of Big Biological Data. Molecular Plant 13:1194-1202. 10.1016/j.molp.2020.06.009

Choudhury D, Sahu JK, Sharma G. 2012. Bamboo shoot: Microbiology, Biochemistry and Technology of fermentation-a review.

De Grassi A, Lanave C, Saccone C. 2008. Genome duplication and gene-family evolution: the case of three OXPHOS gene families. Gene 421:1-6. 10.1016/j.gene.2008.05.011

Gao C, Deng M, Yang X, Yu W, Cai J, Shi Y, Zhu Z, Zhou T, Xue L, Cao F, Wang G, Fu F-F. 2020. Genome-Wide Identification and Coexpression Network Analysis of DNA Methylation Pathway Genes and Their Differentiated Functions in Ginkgo biloba L. Forests 11:1076. 10.3390/f11101076

Guo ZH, Ma PF, Yang GQ, Hu JY, Liu YL, Xia EH, Zhong MC, Zhao L, Sun GL, Xu YX, Zhao YJ, Zhang YC, Zhang YX, Zhang XM, Zhou MY, Guo Y, Guo C, Liu JX, Ye XY, Chen YM, Yang Y, Han B, Lin CS, Lu Y, Li DZ. 2019. Genome Sequences Provide Insights 
431

432

433

434

435

436

437

438

439

440

441

442

443

444

445

446

447

448

449

450

451

452

453

454

455

456

457

458

459

460

461

462

463

464

465

466

467

468

469

470

471

472

473

474

475

476

into the Reticulate Origin and Unique Traits of Woody Bamboos. Molecular Plant 12:1353-1365. 10.1016/j.molp.2019.05.009

Kahle KT, Rinehart J, Ring A, Gimenez I, Gamba G, Hebert SC, Lifton RP. 2006. WNK protein kinases modulate cellular $\mathrm{Cl}-$ flux by altering the phosphorylation state of the Na-K-Cl and K-Cl cotransporters. Physiology 21:326-335. 10.1152/physiol.00015.2006

Kumar K, Raina SK, Sultan SM. 2020. Arabidopsis MAPK signaling pathways and their cross talks in abiotic stress response. Journal of Plant Biochemistry and Biotechnology 29:700714. 10.1007/s13562-020-00596-3

Kumar K, Rao KP, Biswas DK, Sinha AK. 2011. Rice WNK1 is regulated by abiotic stress and involved in internal circadian rhythm. Plant Signaling Behavior 6:316-320. 10.4161/psb.6.3.13063

Kumar S, Stecher G, Li M, Knyaz C, Tamura K. 2018. MEGA X: Molecular Evolutionary Genetics Analysis across Computing Platforms. Molecular Biology and Evolution 35:1547-1549. 10.1093/molbev/msy096

Kundan Kumar, Manu Kumar, Seong-Ryong Kim, Hojin Ryu, Cho Y-G. 2013. Insights into genomics of salt stress response in rice. Rice 6. 10.1186/1939-8433-6-27

Li X, Li J, Cai M, Zheng H, Cheng Z, Gao J. 2020. Identification and Evolution of the WUSCHELRelated Homeobox Protein Family in Bambusoideae. Biomolecules 10. 10.3390/biom10050739

Liu J, Cheng Z, Xie L, Li X, Gao J. 2019. Multifaceted Role of PheDof12-1 in the Regulation of Flowering Time and Abiotic Stress Responses in Moso Bamboo (Phyllostachys edulis). International Journal of Molecular Sciences 20. 10.3390/ijms20020424

Manuka R, Karle SB, Kumar K. 2019. OsWNK9 mitigates salt and drought stress effects through induced antioxidant systems in Arabidopsis. Plant Physiology Reports 24:168-181. 10.1007/s40502-019-00448-w

Manuka R, Saddhe AA, Kumar K. 2015. Genome-wide identification and expression analysis of WNK kinase gene family in rice. Computational Biology and Chemistry 59 Pt A:56-66. 10.1016/j.compbiolchem.2015.09.003

Manuka R, Saddhe AA, Kumar K. 2018. Expression of OsWNK9 in Arabidopsis conferred tolerance to salt and drought stress. Plant Science 270:58-71. 10.1016/j.plantsci.2018.02.008

Manuka R, Saddhe AA, Srivastava AK, Kumar K, Penna S. 2021. Overexpression of rice OsWNK9 promotes arsenite tolerance in transgenic Arabidopsis plants. Journal of Biotechnology 332:114-125. 10.1016/j.jbiotec.2021.04.001

McCormick JA, and Ellison DH. 2011. The WNKs: atypical protein kinases with pleiotropic actions. Physiological Reviews 91:177-219. 10.1152/physrev.00017.2010

Nakamichi N, Murakami-Kojima M, Sato E, Kishi Y, Yamashino T, Mizuno T. 2002. Compilation and characterization of a novel WNK family of protein kinases in Arabiodpsis thaliana with reference to circadian rhythms. Bioscience, biotechnology, and biochemistry. 66:2429-2436.

Ramakrishnan M, Yrjälä K, Vinod KK, Sharma A, Cho J, Satheesh V, Zhou M. 2020. Genetics and genomics of moso bamboo (Phyllostachys edulis): Current status, future challenges, and biotechnological opportunities toward a sustainable bamboo industry. Food and Energy Security 9:e229. 10.1002/fes3.229

Rodan AR, and Jenny A. 2017. WNK Kinases in Development and Disease. Current Topics in Developmental Biology 123:1-47. 10.1016/bs.ctdb.2016.08.004 
477 Urano D, Czarnecki O, Wang X, Jones AM, Chen JG. 2015. Arabidopsis receptor of activated C $478 \quad$ kinase 1 phosphorylation by WITH NO LYSINE8 KINASE. Plant Physiology 167:507-

479

480

481

482

483

484

485

486

487

488

489

490

491

492

493

494

495

496

497

498

499

500

501

502

503

504

505

506

507

508

509

510

511

512

513

514

515

516

517

518

519

520

521
516. 10.1104/pp.114.247460

Urano D, Phan N, Jones JC, Yang J, Huang J, Grigston J, Taylor JP, Jones AM. 2012. Endocytosis of the seven-transmembrane RGS1 protein activates G-protein-coupled signalling in Arabidopsis. Nature Cell Biology 14:1079-1088. 10.1038/ncb2568

Verõ Âssimo F, and Jordan P. 2001. WNK kinases, a novel protein kinase subfamily in multicellular organisms. Oncogene 20:5562-5569. 10.1038/sj.onc.1204726

Wang D, Cao Z, Wang W, Zhu W, Hao X, Fang Z, Liu S, Wang X, Zhao C, Tang Y. 2020. Genome-Wide Characterization of OFP Family Genes in Wheat (Triticum aestivum L.) Reveals That TaOPF29a-A Promotes Drought Tolerance. Biomed Research International 2020:9708324. 10.1155/2020/9708324

Wang Y, Liu K, Liao H, Zhuang C, Ma H, Yan X. 2008. The plant WNK gene family and regulation of flowering time in Arabidopsis. Plant Biology 10:548-562. 10.1111/j.14388677.2008.00072.x

Wang Y, Suo H, Zheng Y, Liu K, Zhuang C, Kahle KT, Ma H, Yan X. 2010. The soybean rootspecific protein kinase GmWNK1 regulates stress-responsive ABA signaling on the root system architecture. Plant Journal 64:230-242. 10.1111/j.1365-313X.2010.04320.x

Wang Y, Suo H, Zhuang C, Ma H, Yan X. 2011. Overexpression of the soybean GmWNK1 altered the sensitivity to salt and osmotic stress in Arabidopsis. Journal of Plant Physiology 168:2260-2267. 10.1016/j.jplph.2011.07.014

Xie M, Wu D, Duan G, Wang L, He R, Li X, Tang D, Zhao X, Liu X. 2014. AtWNK9 is regulated by $\mathrm{ABA}$ and dehydration and is involved in drought tolerance in Arabidopsis. Plant Physiology and Biochemistry 77:73-83. 10.1016/j.plaphy.2014.01.022

Xu B-e, English JM, Wilsbacher JL, Stippec S, Goldsmith EJ, Cobb MH. 2000. WNK1, a novel mammalian serine/threonine protein kinase lacking the catalytic lysine in subdomain II. Journal of Biological Chemistry 275:16795-16801.

$\mathrm{Xu}$ J, and Zhang S. 2015. Mitogen-activated protein kinase cascades in signaling plant growth and development. Trends in Plant Science 20:56-64. 10.1016/j.tplants.2014.10.001

Yang W, Lu Z, Xiong Y, Yao J. 2017. Genome-wide identification and co-expression network analysis of the OsNF-Y gene family in rice. The Crop Journal 5:21-31. 10.1016/j.cj.2016.06.014

Yang Y, Yewei H, Shuping L, Li Y, Zhijun Z. 2010. Effects of $\mathrm{NaCl}$ stress on chlorophyll fluorescence and physiologi cal characteristics of moso bamboo seedlings. Journal of Bamboo Research 29:29-32.

Yuan Y, Fang L, Karungo SK, Zhang L, Gao Y, Li S, Xin H. 2016. Overexpression of VaPAT1, a GRAS transcription factor from Vitis amurensis, confers abiotic stress tolerance in Arabidopsis. Plant Cell Reports 35:655-666. 10.1007/s00299-015-1910-x

Zhang B, Liu K, Zheng Y, Wang Y, Wang J, Liao H. 2013. Disruption of AtWNK8 enhances tolerance of Arabidopsis to salt and osmotic stresses via modulating proline content and activities of catalase and peroxidase. International Journal of Molecular Sciences 14:7032-7047. 10.3390/ijms14047032

Zhao H, Gao Z, Wang L, Wang J, Wang S, Fei B, Chen C, Shi C, Liu X, Zhang H, Lou Y. 2018. Chromosome-level reference genome and alternative splicing atlas of moso bamboo (Phyllostachys edulis). Gigascience 7:giy115. 10.1093/gigascience/giy115 
522 Zhao J, Gao P, Li C, Lin X, Guo X, Liu S. 2019. PhePEBP family genes regulated by plant 523 hormones and drought are associated with the activation of lateral buds and seedling 


\section{Figure 1}

The phylogenetic tree of WNK genes from dicot and monocot plants.

The phylogenetic tree was constructed using WNK sequences of Arabidopsis thaliana (At), Glycine max (Gm), Oryza sativa (Os), Zea mays (Zm), P. edulis (Pe), O. latifolia (Ola), R. guianensis (Rgu), G. angustifolia (Gan) and B. amplexicaulis (Bam). The bootstrap support values were mentioned as the numbers on the branches. Clade I, II and III are indicated in the blue, violet and pink colours, respectively. The dicot plants WNK genes were indicated in the grey colour boxes and the diploid bamboo species are indicated in the yellow colour boxes. The conserved motifs (1-10) are mentioned in different colour boxes. 


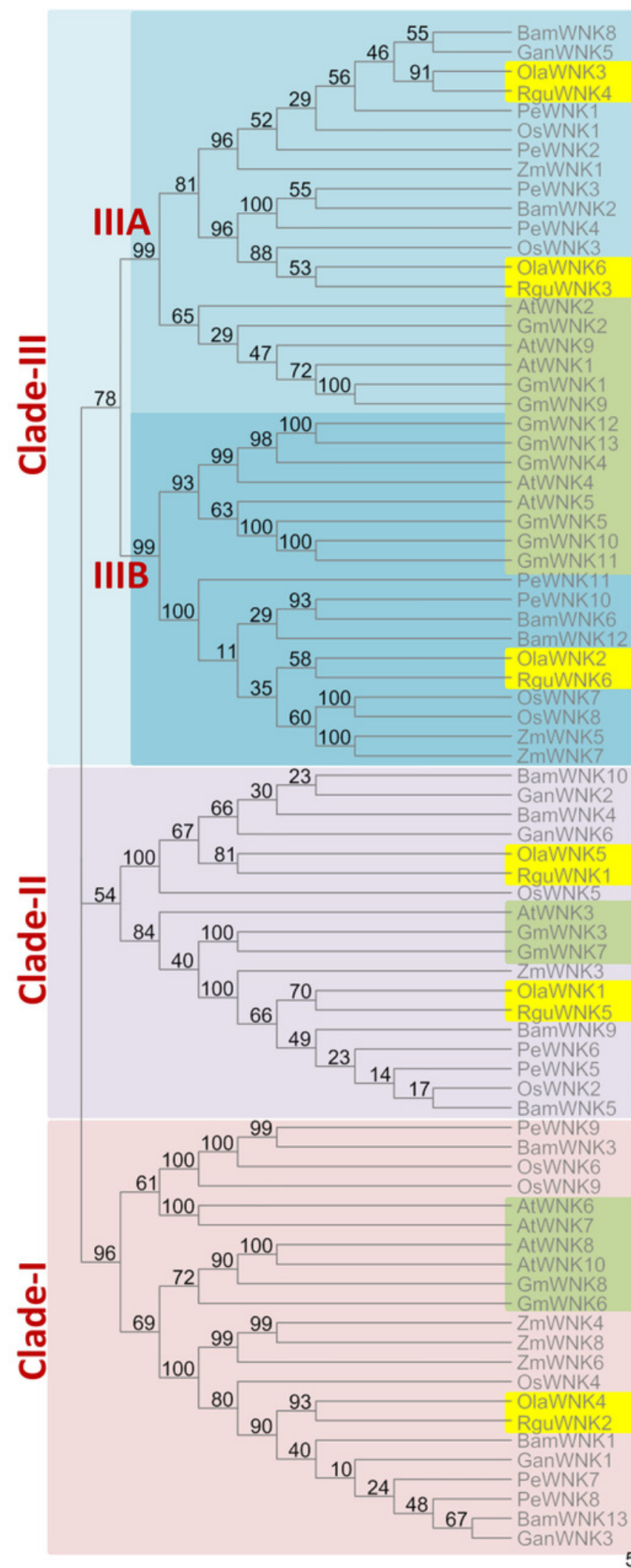

\begin{tabular}{|c|c|}
\hline$-94 \div 6-3-1-8<2-5$ & Motif 9 \\
\hline$-4-3-1-8 / 2-5-7 / 10$ & Motif 4 \\
\hline $94,6-3=1-82-5-7 / 10-$ & Motif 6 \\
\hline$-46-3-1-82-5-7 / 10$ & \\
\hline$-9 / 4: 6-3 / 7-8 / 2-5$ & Motif 3 \\
\hline$-9 / 4: 3-3 / 8 / 2-5$ & Motif 1 \\
\hline $94: 6-3 \cdot 1-8: 2-5$ & Motif 2 \\
\hline$-9 / 4: 6-3: 1-8: 2-5-7 / 10-$ & Motif 5 \\
\hline $94: 6-3: 1-8: 2-5-710$ & Motif 7 \\
\hline 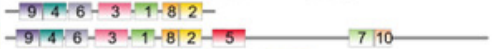 & Motif 10 \\
\hline
\end{tabular}

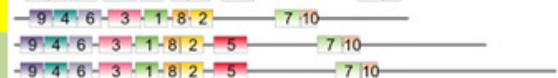

$-3-1-8 / 2-5-7 / 10-$

$9 \times 4 \cdot 6-3 \cdot 1-8 / 2-5-7 / 10-710$

$-9446-3=1-8 / 2-5-7 / 10-$

9. $4: 6-3=1-8 / 2-5-710$

$-4 \cdot 6-3-1-8 / 2-5-7 / 10-$

4.6-1-8:2-5-7.10-

4- $1-822-7,10-7.2$

$74: 6-3 \times 1-8 / 2-5-7: 10-$

$94 \div 6-3-7-8 / 2-5-710$

$-9 \times 4: 6-3: 5 \cdot 8 / 2-5-7 / 10-$

$-9 / 4: 3-3: 1-8 / 2-5-7 / 10$

$4 \cdot 6-3-1-8 / 2-5-$

$-9: 4: 6-3: 7 \times 8: 2-5-7 / 10-$

$-94 \times 6-3-1-82-5-710$

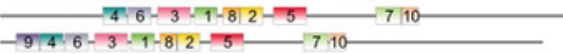

$4: 6-3=1-8 / 2-5-7 / 10-$

4:6-3 $37-8 / 2-5-7 / 10-$

$-906-3-7-82-5-710$

$-946-3 \sqrt{4}-8 / 2-5-7 / 10$

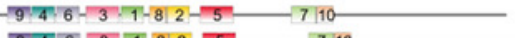

$-9466-3 \cdot 1-8 / 2-5-7 / 10$

$-9,4: 6-3-7-8 / 2-75-7 / 10$

$9 / 4-6-3-1-8 \div 2-5$

$94-6-3,1-822-5$

$9 \sqrt{9} 6-3-3-8,2-5$

$9,4 \div 6-3-1-82-5$

$-946-317-82-5$

$6-3-1-82-5-$

-94.6-3 $1-82-5$

$94: 6-3: 17-82-5-710$

$-9 \times 4: 6-3=1-82-5-710-$

$-9 \sqrt{4}: 6-3 \cup 1-8 / 2-5-710-$

$902-4 \cdot 3-3 \cdot 1-8 \cdot 2-5$

$-4: 6-3=1-8 / 2-5-710-$

$-9-9-4: 6-3-1-8 / 2-5-7 / 10$

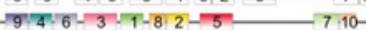

-9:4: $6-3-17-8 / 2-5-7 / 10-7 / 10$

$94-6-3-1-32-5$

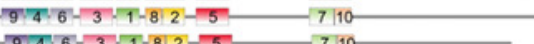

$-9 / 4: 6-3: 1-8 / 2-5-7 / 10$

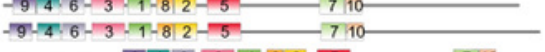

$9406-349-82-5-7 / 10-$

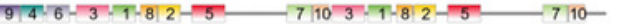

$-9 / 4: 6-3 \cdot 9-8 \div 2-5-7 / 10-$

$-9,4: 6-3 \times 1: 8: 2-5-710-$

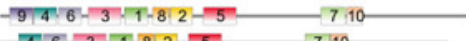

$-4 \cdot 6-3-1+8: 2-5-7110-710$

$-4.6-3$
$-4.6-3$

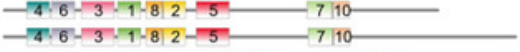

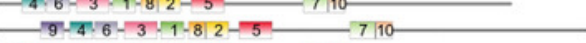

- $4: 6-3=1-82-5-$

$46-347-8: 5-710$

-9.4. $6-3 \times 1-8 / 2-5$

$-7 \cdot 10$

$1.8+2-5-7 \mid 10-$

$94 \cdot 6-2-5-710-710$

$-9,4: 6-3 \cdot 1-8,2-5-710-70$

$-9.4: 6-8 / 2-5-7 / 10-$

$-94 / 6-3 / 1-8 / 2-5-7 / 10-$

$-9046-3,9-82-5-7: 10$

$-4 \cdot 6-3 \cdot 9 \cdot 2-5-710-$

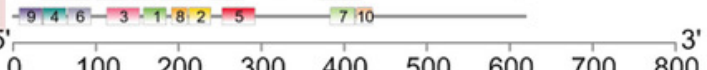


Figure 2

The conserved cis-elements analysis of WNK genes in the promoter regions of Bambusoideae and other monocot and dicot plants, related to stress response (P-box, ABRE, GARE-motif, TCA and circadian). 


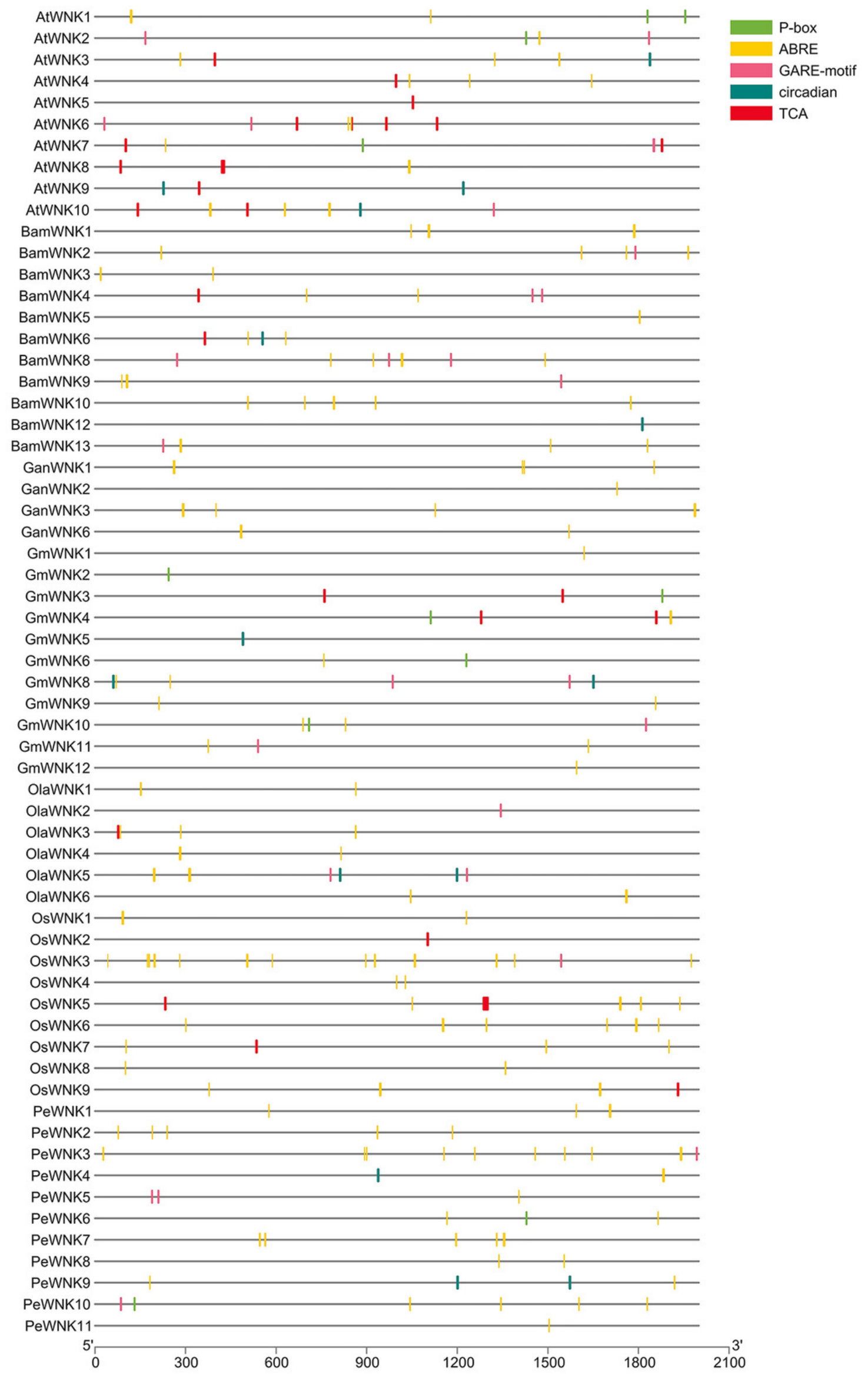

PeerJ reviewing PDF | (2021:08:65078:2:0:NEW 25 Nov 2021) 
Figure 3

Multiple sequence alignment between PeWNK1, HUMAN_WNK1, GnWNK1, OsWNK9, and AtWNK1 protein sequences. Conserved domains, motif and secondary structural arrangements were highlighted. The phosphorylation sites were mentioned in the blue background. 
PeWNK1 HUMAN WNK 3

GnWNK1

OsWNK9

AtWNK1

consensus $>70$

Loop region

PeWNK1 HUMAN_WNK3 GnWNK1

OsWNK9

AtWNK1

consensus $>70$

Loop region
PeWNK1

HUMAN_WNK3

GnWNK1

OsWNK9

AtWNK1

consensus $>70$

Loop region

PeWNK1 HUMAN_WNK3

GnWNK1

OsWNK9

AtWNK1

consensus $>70$

Loop region

PeWNK1

HUMAN_WNK3

GnWNK1

OsWNK9

AtWNK1

consensus $>70$

Loop region
58

181

58

58

... MMGANTNAADCPEYAEVDRTGRYGRYNDVLGKGASKTVYRAFDEYQGMEVAWNQVKI 121 FKEKNEKEMEEEAEMKAVATSPSGRF LKF IELGRGAFKTVYKGLDTE TWVEVAWCELQD 1 ...MYKGRFGGKAELGYVET DPSGRYGRFRDILGKGAVKVVYRAFDEVLGREVAWNQVKI 6 AEAEEQPPDKDGDEEGYVEADPAGRFIRYDEIVGSGAVKTVYKAFDKLEGVEVAWSQSRI 1 ... MNNLSYLEPDYSEEVEVDPTGRYGRYNEV LGKGASKTVYRAFDEYEGIEVAWNQVKL

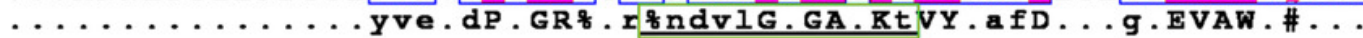

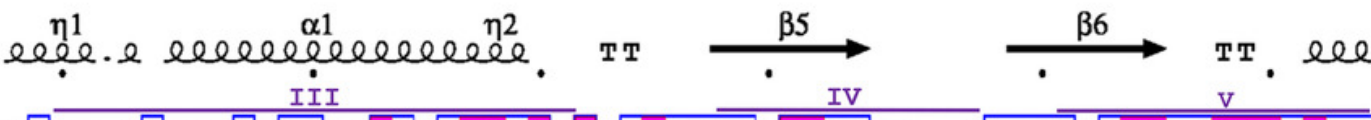
HD $\overline{\text { L . QSSPEDIER RYCEIHLLKTLKHR NIMKFYTSWVDIS . RRN INFITEMFTSGTLRQ }}$

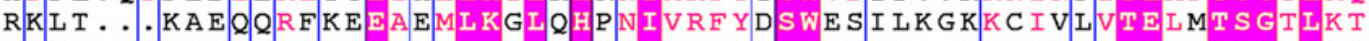

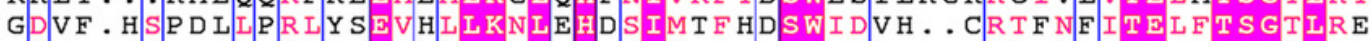
DDSVMGSSKKMKQLNTEIQLLKTLKHKNIEKMF ASWVDE E . KKTVNIITELFTSGSLTQ Y D F L QS P E DLER L Y CEI HLLKTLKHKNIMKFYTSWVDTA . NRN INFVT EIFT S GTLRO

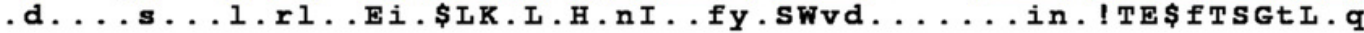

$\alpha 2$

elel

$\alpha 3$

elecelecelecele
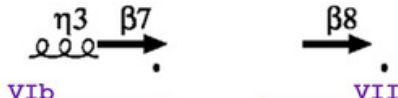

115

YRQRHR R N I W T VKH NCRQ I LSGLLYLHSHD P I I HRD L KCDN I EVNGN Y L K R F K VMK P KVLRSWCRQ I LKG L Q F L HTRTP P I I HRD L K CDN I F I T GP T GSVK I GD L G L YRKKYQR VDIRA VKNWARQ I LSG LEY L HS HDP P VI HRD L K CDN I F I NGH L GQVK I GD L G I

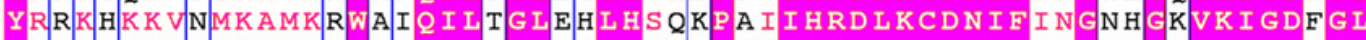
YR L R H K R VN I RAMKHWCRQ I LRG L HY L HS HDP P VI HRD L K C D N I EVNGN Q GEVK I G D L G L

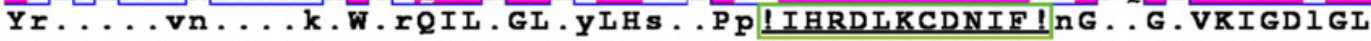

175

298

175

184

175

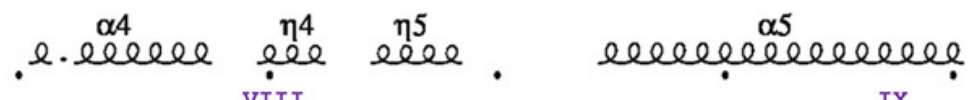

AAILRKS. HAVHCVGTPEFMAP EVYEEEYNELVDIYSFGMCVLEMVTFEYPYSECTNPVQ ATLMRTS. FAKSVIGTPEFMAP EMYEEHYDESVDVYAF GMCMLEMATSEYPYSECQNAAQ A A IRG S $Q$ H H SVIG TPEFMAP ELYEEEYNELVDIYSF GMCMIEIFTSEFPYSECSNPAQ

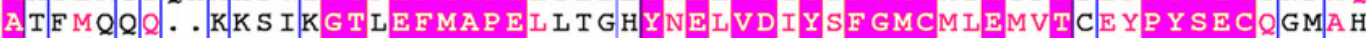
AAI LRKS. H A A H CVG TPEFMAP EVYEEAYNELVDIY SF GMCI LEMVTF DYPYSECTHP AO

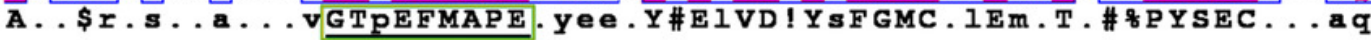

eber. 234 357

IYKKVISGTRPEAL YKVKDL MVRQ TVEKCLA. TASRRLPARELLKDPFIQTDDVPFCSGD IYRKVISSGIKPASFNKVTDP EVKEIIEGCIRQNKSERLS IRDL LNHAFF A E T G LRVELA I YKKVTSGKLPEAYYRIHDLEAQKFVGKCSA. NVSERLSAKELLLDPFIATEQLDSPLPS IFKKIDEGKKPAAF YKI KDAEVRSF I ENCLA. PVENRMS A T EL LKS SE LQDDD L I SVS LV

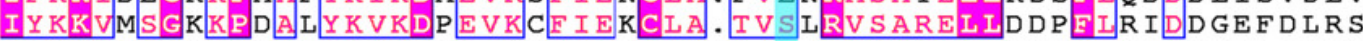

I qkK!.sG.kP.a.yk!.D.ev..f!e.C.a...s.RIsa.\#LL...FI..\#d.....

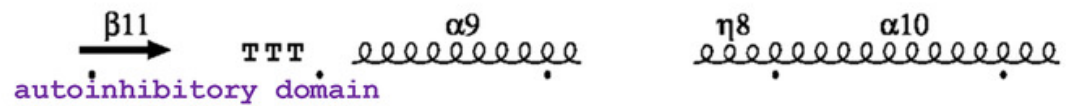

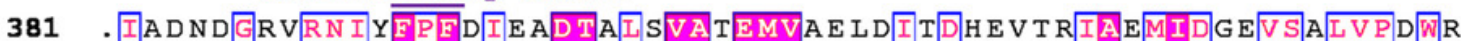

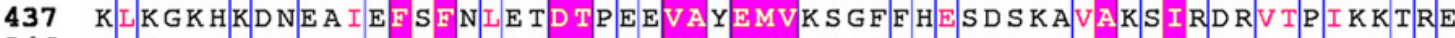
346 . IS NKNG $Q K R$ R I F F P F D T I NDTA I DVAMEMVKE L E I S D L E P L E I A E M I E E E I S A L V P T W R

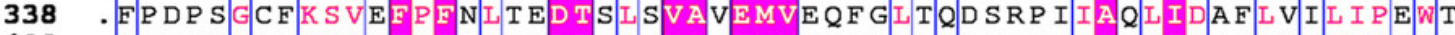

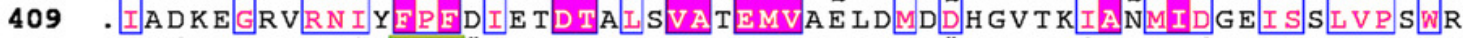
...d..g...! . F PF\#...DT...VA.EMV.e...\#....!AemId..v..Ivp.w. 
Figure 4

Conserved domain sequence analysis of WNK in the Bambusoideae (PeWNK1) protein predicted by Consurf server.

Predicted homology model of PeWNK1 using modeler; highly conserved WNK kinase domain and autoinhibitory domain were highlighted.

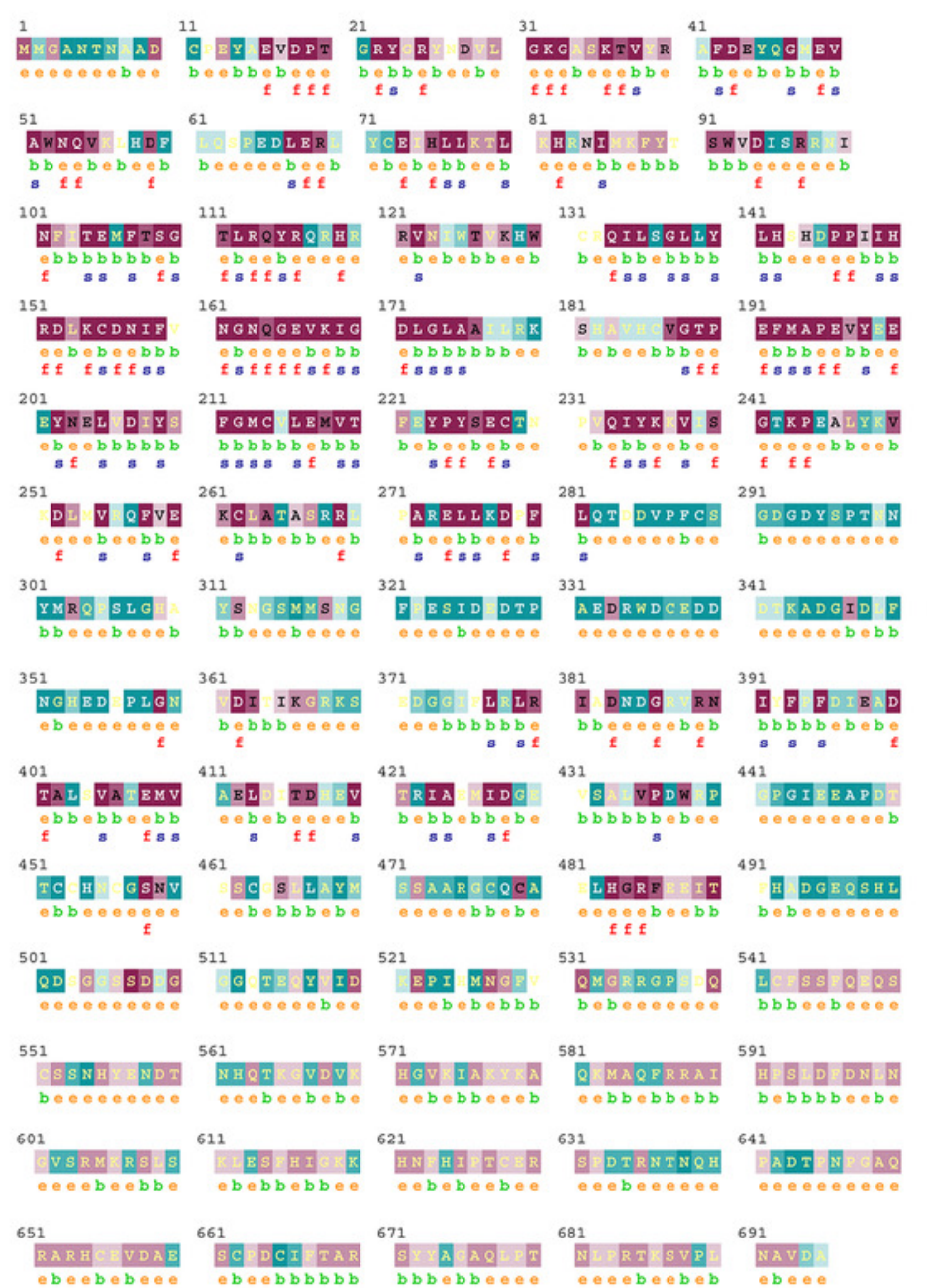

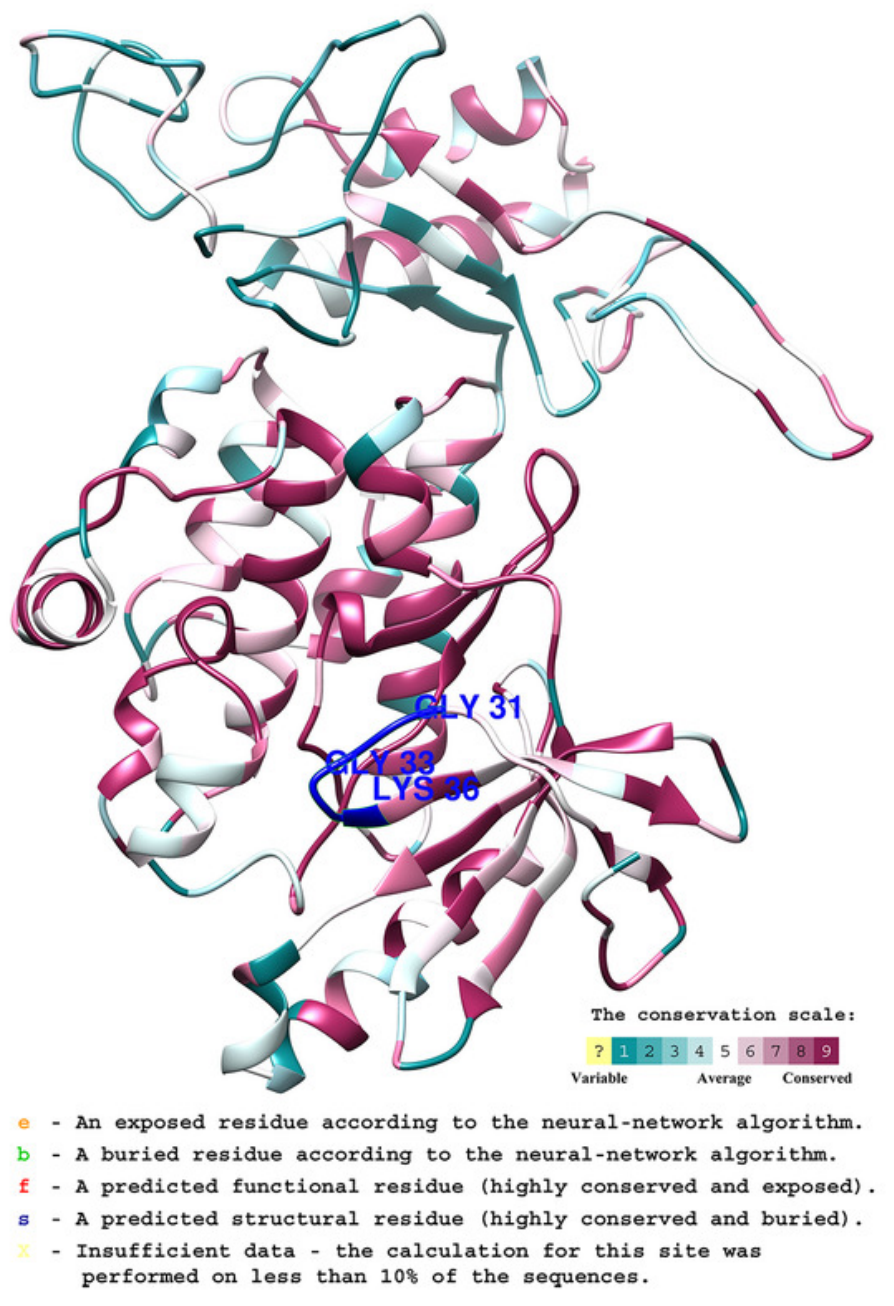


Figure 5

Expression analysis of PeWNK genes for the circadian cycle.

qRT-PCR analysis of PeWNK5, PeWNK7, PeWNK8 and PeWNK11 genes normalized with NTB. Moso bamboo cDNA leaf samples 0-48 $\mathrm{h}$. The error bar indicates the standard deviation $(n=3)$.

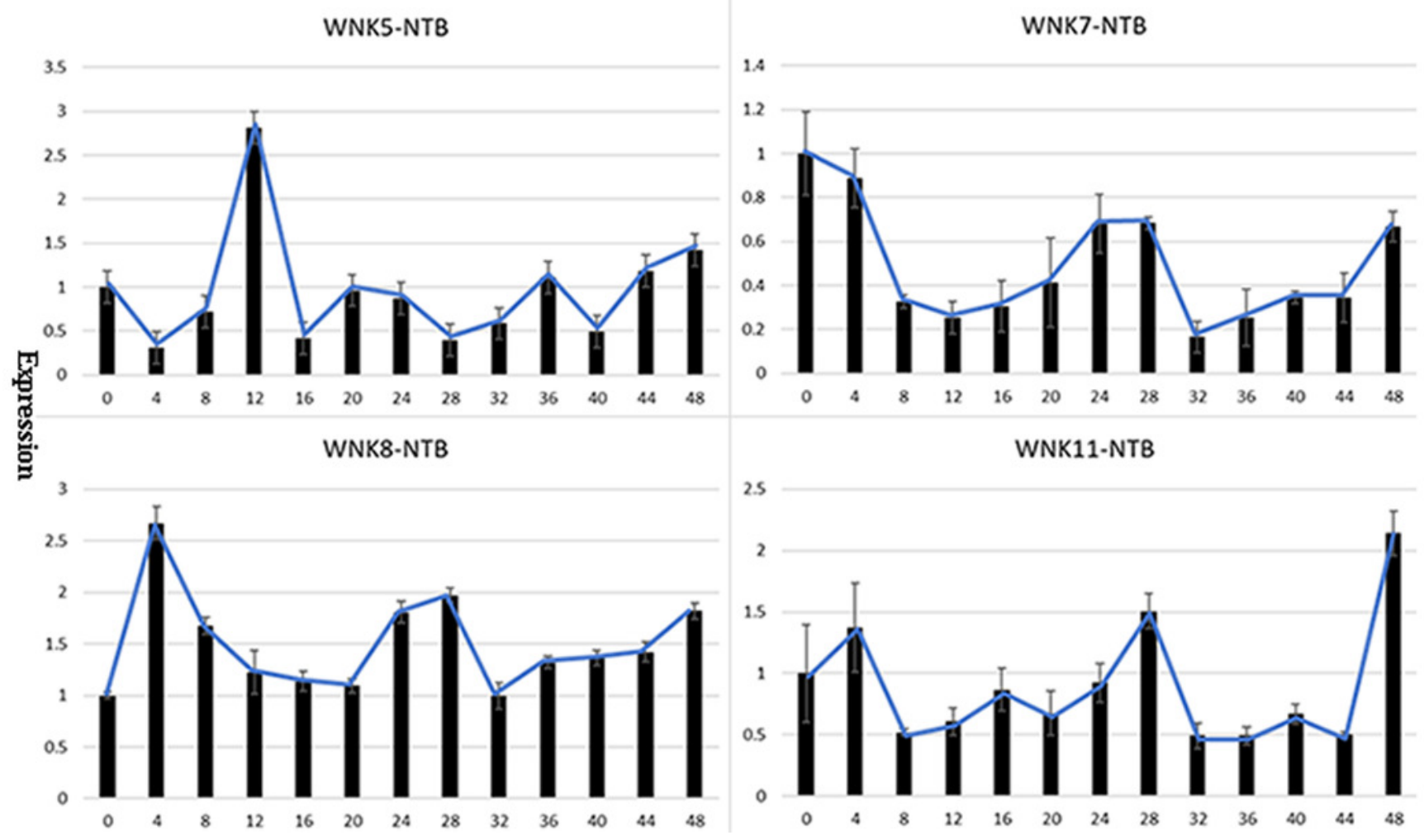


Figure 6

Expression of PeWNK genes in 26 different tissues and stages of bamboo growth. The log2 expression values represent each colour box and the colour scale is present on the upper right side.

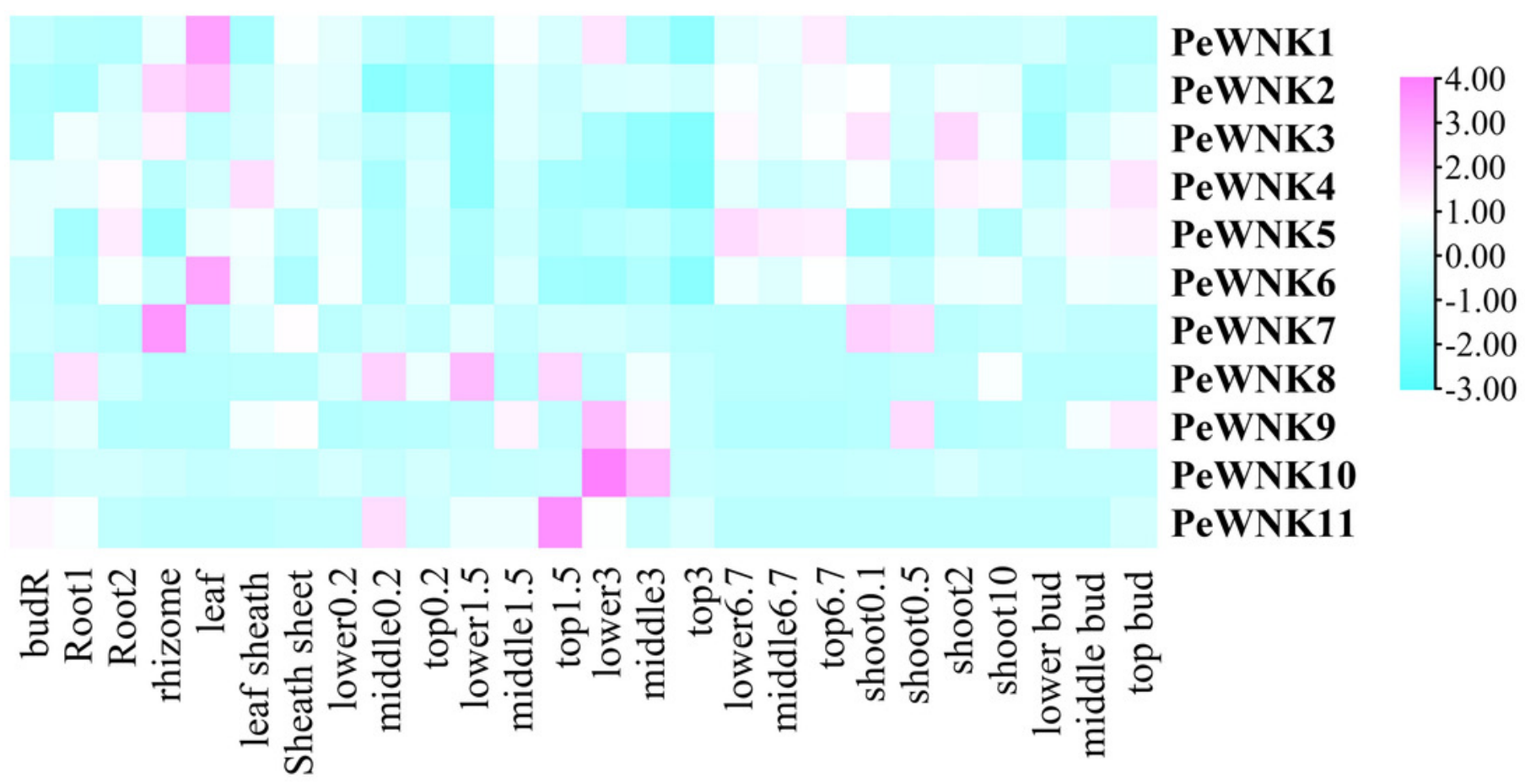




\section{Figure 7}

Expression analysis of PeWNK genes in response to Polyethylene glycol (PEG), Sodium chloride ( $\mathrm{NaCl})$, Abscisic acid (ABA) and Salicylic acid (SA).

The FPKM values of transcriptomic data (Moso bamboo seedlings treated with PEG (25\%), $\mathrm{NaCl}(200 \mathrm{mM}), \mathrm{ABA}(1 \mathrm{uM}), \mathrm{SA}(1 \mathrm{mM})$ for $3 \mathrm{~h}$ and $24 \mathrm{~h}$ ) are used to develop graphs. The error bar indicates the standard deviation $(n=3)$. 

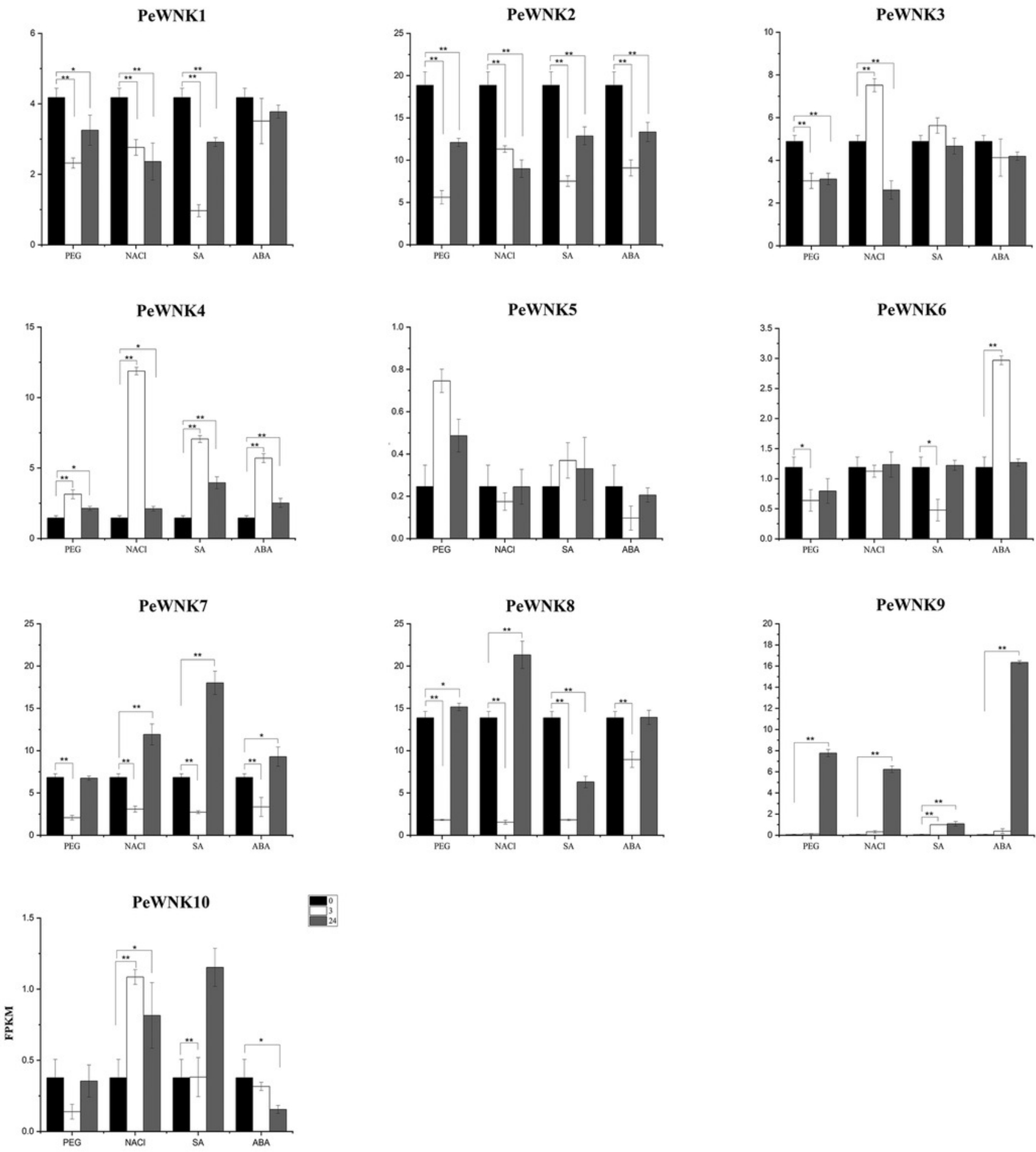


\section{Figure 8}

Co-expression network of PeWNK8 (PH02Gene03413.t1), PeWNK2 (PH02Gene17877), PeWNK4 (PH02Gene23702), PeWNK7 (PH02Gene03314). The boxes indicate the genes involved in abiotic stress response.

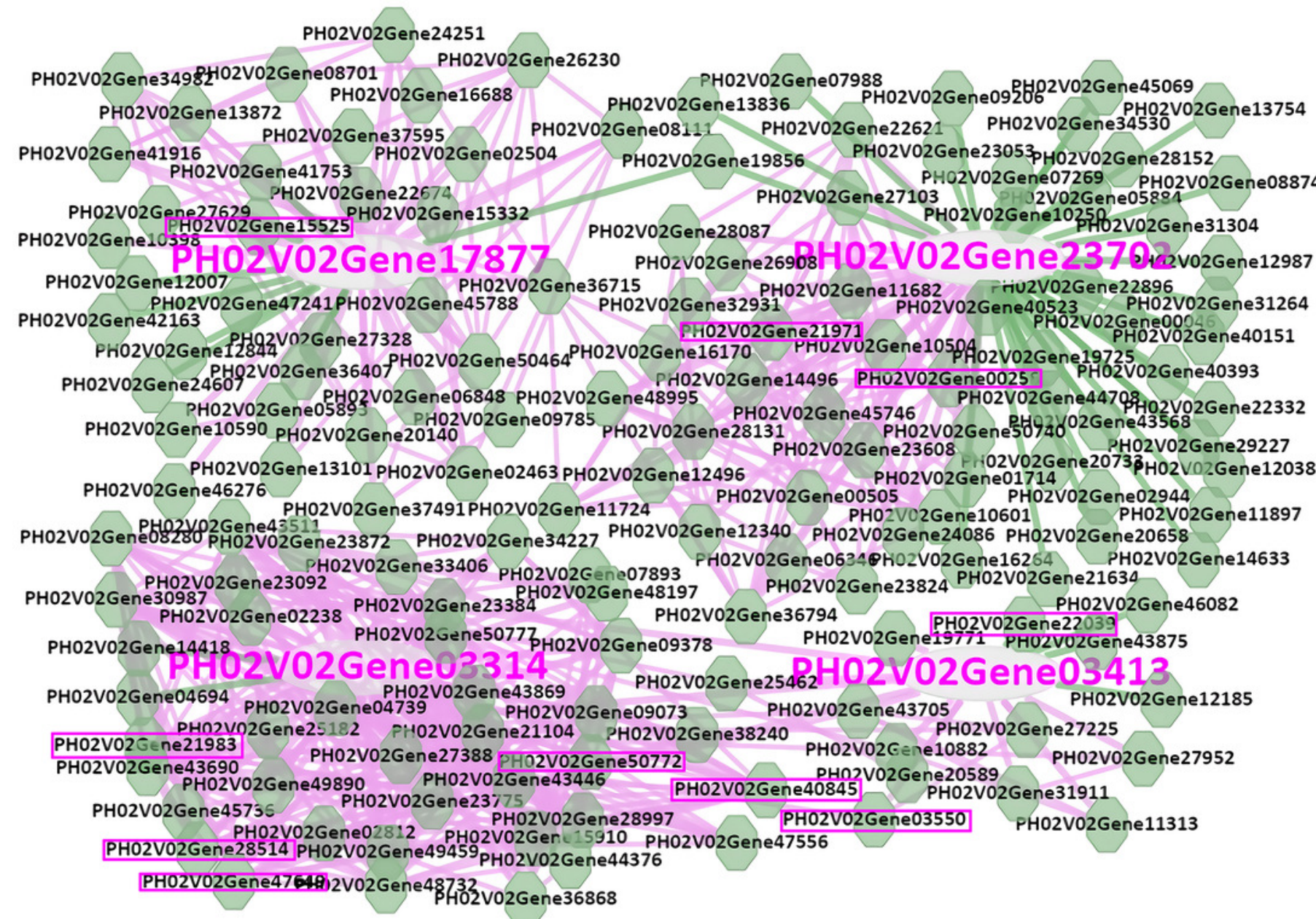


Table $\mathbf{1}$ (on next page)

Protein secondary structure of WNK protein in P. edulis 
3 Table. 1. Protein secondary structure of WNK protein in P. edulis

\begin{tabular}{lccccc}
\hline Protein & ID of gene & Alpha helix & Beta turn & Extended strand & Random coil \\
\hline PeWNK1 & PH02Gene37861.t1 & $36.12 \%$ & $3.74 \%$ & $8.92 \%$ & $51.22 \%$ \\
PeWNK2 & PH02Gene17877.t1 & $37.07 \%$ & $5.75 \%$ & $11.65 \%$ & $45.54 \%$ \\
PeWNK3 & PH02Gene03314.t1 & $41.44 \%$ & $3.75 \%$ & $9.30 \%$ & $45.51 \%$ \\
PeWNK4 & PH02Gene01510.t1 & $38.27 \%$ & $3.91 \%$ & $10.10 \%$ & $47.72 \%$ \\
PeWNK5 & PH02Gene07448.t1 & $37.37 \%$ & $5.30 \%$ & $11.65 \%$ & $45.69 \%$ \\
PeWNK6 & PH02Gene25768.t1 & $38.12 \%$ & $4.06 \%$ & $8.59 \%$ & $49.22 \%$ \\
PeWNK7 & PH02Gene38251.t1 & $37.69 \%$ & $3.90 \%$ & $9.85 \%$ & $48.56 \%$ \\
PeWNK8 & PH02Gene03413.t1 & $35.49 \%$ & $4.57 \%$ & $11.04 \%$ & $48.90 \%$ \\
PeWNK9 & PH02Gene20314.t1 & $42.60 \%$ & $5.47 \%$ & $10.95 \%$ & $40.98 \%$ \\
PeWNK10 & PH02Gene23702.t1 & $40.21 \%$ & $3.96 \%$ & $10.83 \%$ & $45.00 \%$ \\
PeWNK11 & PH02Gene11468.t1 & $36.32 \%$ & $6.21 \%$ & $16.32 \%$ & $41.15 \%$ \\
\hline
\end{tabular}

4 